\title{
A Multi Center Experience: Is Valve Replacement Safe for Patients with Hugely Dilated Left Ventricle?
}

\author{
Ahmed Ahmed, MD, ${ }^{1}$ Ahmed Toema, $M D,{ }^{2}$ Ahmed Yehia, $M D,{ }^{2}$ Yassin Hashim, MD ${ }^{2}$ \\ Elkahely Mohamed, MD, ${ }^{3}$ Ahmed Onsy, MD ${ }^{4}$ \\ ${ }^{1}$ Cardiothoracic Surgery Department, Faculty of Medicine, Ain Shams University, Cairo, Egypt; ${ }^{2}$ Cardiothoracic Surgery \\ Department, Faculty of Medicine, Helwan University, Cairo, Egypt; ${ }^{3}$ Cardiothoracic Surgery Department, Faculty of Medicine, Al \\ Azhar University, Cairo, Egypt; ${ }^{4}$ Cardiology Department, Faculty of Medicine, Ain Shams University, Cairo, Egypt
}

\section{ABSTRACT}

Background: Dilated left ventricle occurs in chronic aortic and mitral regurgitations. We describe the early outcome of mitral and aortic valve replacement for patients with severely dilated left ventricle in different surgical interventions.

Methods: From March 2014 to December 2018, 620 patients with left ventricular end-diastolic diameter (LVEDD) of $\geq 70 \mathrm{~mm}$ underwent valve replacement procedures in 8 cardiac surgery centers in Egypt. One hundred ninety four cases $(31.3 \%)$ underwent aortic valve replacement, 173 cases $(27.9 \%)$ underwent mitral valve replacement, 123 cases $(19.9 \%)$ underwent double valve replacement, 59 cases $(9.5 \%)$ underwent double valve replacement with either tricuspid valve repair or replacement, 33 cases $(5.3 \%)$ underwent mitral valve replacement with either tricuspid valve repair or replacement, 20 cases $(3.2 \%)$ underwent mitral valve replacement with CABG, 10 cases (1.6\%) underwent aortic valve replacement with CABG, while 8 cases $(1.3 \%)$ underwent aortic valve replacement with ascending aortic aneurysm repair.

Results: Four patients (0.6\%) developed new postoperative renal failure, which required dialysis. Twentynine patients $(4.7 \%)$ required reoperation for bleeding. One patient $(0.2 \%)$ developed sternal dehiscence. Five patients $(0.8 \%)$ postoperatively developed stroke. Twenty-five patients (4\%) died, and the main causes of death were low cardiac output and sepsis with eventual multi-organ failure.

Conclusion: Valve replacement in patients with hugely dilated left ventricle are safe operations with satisfactory outcomes even if combined with other procedures, especially with proper preoperative preparation, intraoperative preservation of posterior mitral leaflet, and meticulous postoperative follow up in the surgical ICU.

Received May 10, 2019; accepted August 29, 2019.

Correspondence: Ahmed Ahmed, Cardiothoracic Surgery Department, Faculty of Medicine, Ain Shams University, Postal Code 11517, Cairo, Egypt; 00201114567874; fax:00201114567874 (e-mail: afouad38@yahoo.com).

\section{INTRODUCTION}

Even though there is increased awareness about the prevention and management of rheumatic heart disease, many cases still are diagnosed worldwide. Dilated left ventricle is part of the natural history of different valvular pathology, mainly chronic aortic and mitral regurgitations. Some cases are asymptomatic until the left ventricle is quite huge, and some cases unfortunately are neglected in the early stages until the left ventricle is dilated too much, particularly in remote areas with lower than average health care standards. Most of these cases have low ejection fraction (EF) with heart failure symptoms and signs. Mitral and aortic valve replacement procedures are indicated in patients with severe mitral and aortic insufficiency and highly dilated ventricles [Wenbin 2002]. In this paper, we describe the early outcome after operating on such cases in different cardiac surgery procedures. The local ethical committee approved the study protocol.

\section{MATERIALS AND METHODS}

From March 2014 to December 2018, 620 patients with left ventricular end-diastolic diameter (LVEDD) of $\geq 70 \mathrm{~mm}$ were included in the study. Inclusion criteria included patients who underwent different valve replacement procedures for rheumatic, degenerative, or ischemic pathology either alone

Table 1. Cardiac centers participated in the study

\begin{tabular}{lcc}
\hline Hospital category & City & Hospital name \\
\hline University & Cairo & Ain Shams University Hospital (Cardiac Academy) \\
University & Cairo & Ain Shams University Specialized Hospital \\
Governmental & Giza & 6th of October Insurance Hospital \\
Military & Cairo & Air Force Specialized Hospital \\
Private & Menofia & Elaraby Hospital \\
Private & Cairo & Aldorrah Heart Care Hospital \\
Private & Cairo & Dar Alfouad Hospital (Nasr City Branch) \\
Private & Helwan & Dar Alshefaa Hospital
\end{tabular}




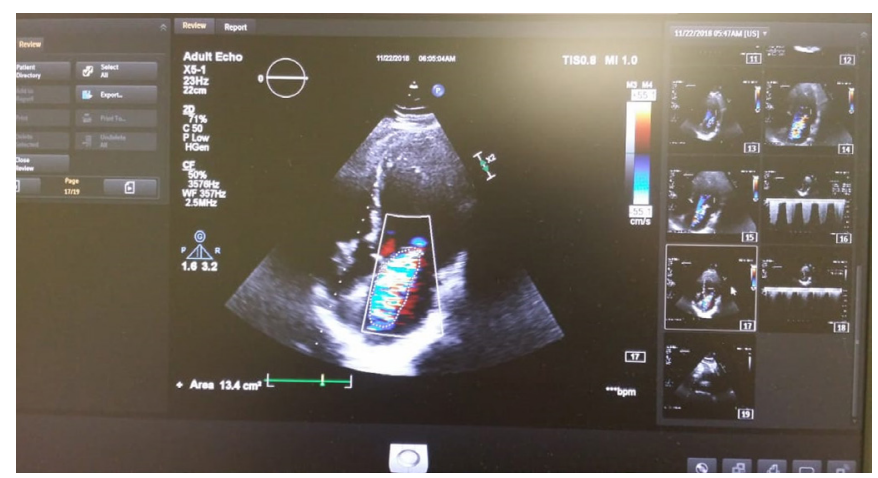

Figure 1. Echocardiography showing severe mitral regurgitation in a patient with hugely dilated left ventricle.

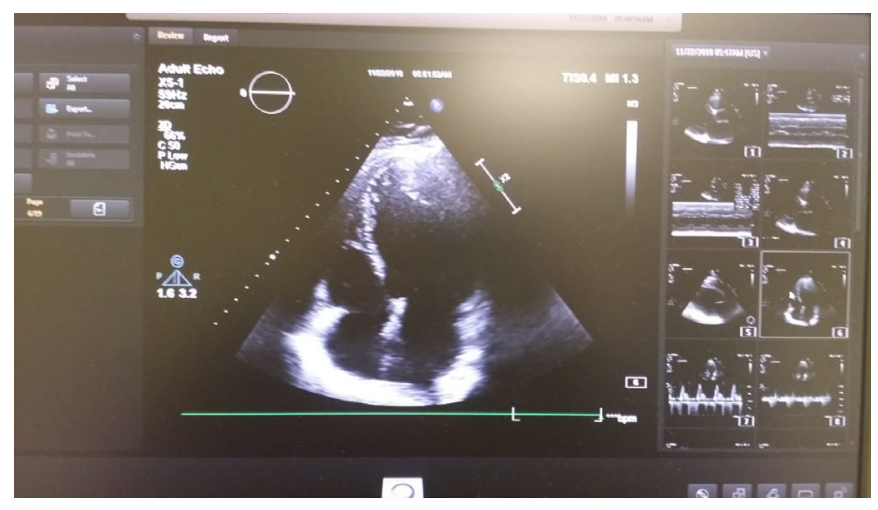

Figure 2. Echocardiography showing severe mitral regurgitation in a patient with hugely dilated left ventricle.

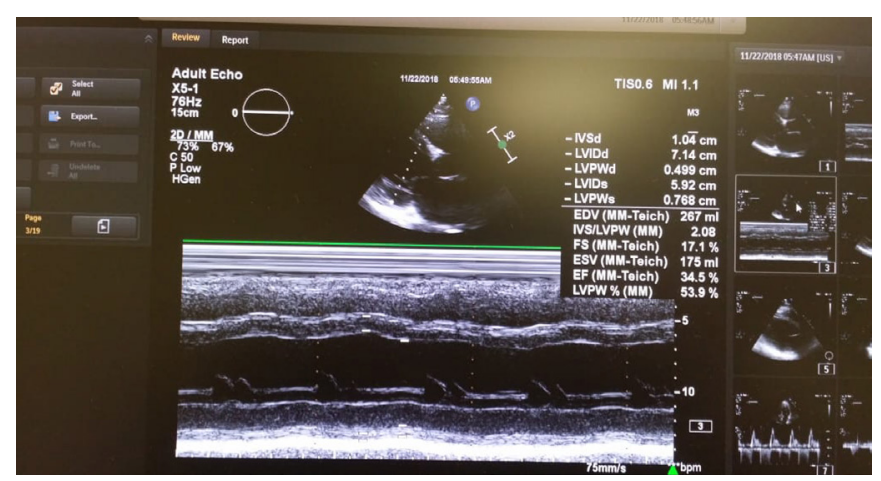

Figure 3. Echocardiography showing severe mitral regurgitation in a patient with hugely dilated left ventricle.

or combined with other cardiac surgical interventions on an elective or urgent basis.

The operations were performed in 8 cardiac surgery centers (Table 1).

Nine patients $(1.5 \%)$ had preoperative serum creatinine of $>2.0 \mathrm{mg} / \mathrm{dL}$, while 2 patients $(0.3 \%)$ were on regular dialysis for chronic renal failure. Erythrocyte sedimentation rate (ESR), C-reactive protein (CRP), and antistreptolysin O (ASO) titer test were measured for patients younger than 25
Table 2. Patient characteristics

\begin{tabular}{lc}
\hline Sex (males) & $416(67.1 \%)$ \\
Age (years) & $17-62(36.9 \pm 12.9)$ \\
Pathology & $549(88.6 \%)$ \\
Rheumatic & $41(6.6 \%)$ \\
Degenerative & $30(4.8 \%)$ \\
Chronic ischemia & \\
NYHA Class & None $(0 \%)$ \\
Class I & $146(23.5 \%)$ \\
Class II & $423(68.3 \%)$ \\
Class III & $51(8.2 \%)$ \\
Class IV & $243(39.2 \%)$ \\
Preop. AF & $20-70(40 \% \pm 13.7)$ \\
EF (\%) &
\end{tabular}

AF: Atrial Fibrillation, EF: Ejection Fraction, NYHA: New York Heart Association, Preop: Preoperative. Description of Data: Age, EF: Range (Mean \pm SD), Sex, NYHA Class, Preop. AF: Number (Percentage)

years to exclude ongoing rheumatic activity. Patients with elevated measurements were referred to cardiology service medical treatment until the markers normalized, and then patients were referred back for surgery. Dobutamine stress echo was performed for patients with EF less than 30\% to confirm the presence of myocardial reserve for valvular cases and myocardial viability for ischemic cases. During the study period, 2 valvular patients and 11 ischemic patients were turned down for operation based on the dobutamine stress echo reports.

The range of the LVEDD by echocardiography was 70-98 $\mathrm{mm}$ (Figures 1, 2, and 3).

Informed consent was taken for the procedure after a detailed explanation of the procedure and possible risk by the surgical team. Permission for data collection was obtained from institutional review boards for the retrograde study, which would not affect the safety or privacy of patients.

All cases were elective except 5 cases $(0.8 \%)$ that were operated on an urgent basis because of associated left main coronary artery disease or unstable angina. All cases routinely were prepared and had the operation on cardiopulmonary bypass with cardioplegic arrest.

Unique protocol was adopted for redo cases, including blood ready in the OR before sternotomy, defibrillation pads on the chest and femoral artery and vein exposure before sternotomy. Although 243 patients (39.2\%) had preoperative atrial fibrillation, concomitant ablation was not performed in order to minimize operation time. Most of these patients will be on warfarin for mechanical valves and because of limitations of resources and experience for arrhythmia surgery in most of the centers, where the procedures were performed. In spite of the fact that patients with rheumatic heart disease often come from limited resource environments, 463 patients $(74.7 \%)$ had mechanical valves. This will require lifelong anticoagulation and monitoring 
Table 3. Operative characteristics

\begin{tabular}{lc} 
Procedure & \\
AVR & $194(31.3 \%)$ \\
MVR & $173(27.9 \%)$ \\
DVR & $123(19.9 \%)$ \\
DVR+TV Repair/TVR & $59(9.5 \%)$ \\
MVR+TV Repair/TVR & $33(5.3 \%)$ \\
MVR+CABG & $20(3.2 \%)$ \\
AVR+CABG & $10(1.6 \%)$ \\
AVR+ Ascending aortic aneurysm & $8(1.3 \%)$ \\
Urgency & \\
Elective & $615(99.2 \%)$ \\
Urgent & $5(0.8 \%)$ \\
Cardioplegia & \\
Cold blood & $578(93.2 \%)$ \\
Custidiol & $42(6.8 \%)$ \\
Aortic Cross-Clamp Time (Minutes) & $49-197$ \\
Bypass Time (Minutes) & $89-242$ \\
Valve Type & \\
Mechanical & $618(99.7 \%)$ \\
Biological & $2(0.3 \%)$ \\
Mitral Leaflet Preservation & $157(25.3 \%)$ \\
Posterior & \\
\hline
\end{tabular}

AVR: Aortic Valve Replacement, CABG: Coronary Artery Bypass Grafting, DVR: Double Valve Replacement, MVR: Mitral Valve Replacement, TV: Tricuspid Valve, TVR: Tricuspid Valve Replacement. Description of Data: Procedure, Urgency, Cardioplegia, Valve type, Mitral leaflet preservation: Number (Percentage), Aortic cross clamp time, Bypass time: Range

based on the relatively young age group to avoid redo surgery in case of biological valves. Operative characteristics are listed in Table 3.

\section{RESULTS}

One hundred nineteen patients (19.2\%) did not require any postoperative inotropic support, while 501 patients $(80.8 \%)$ required inotropic support. Thirteen patients $(2.1 \%)$ required intra-aortic balloon pump (IABP) before induction of anesthesia or after the procedure.

Eighty-three patients (13.4\%) developed new postoperative atrial fibrillation, while 5 patients $(0.8 \%)$ developed complete heart block, requiring insertion of a permanent pacemaker.

Four patients $(0.6 \%)$ developed new postoperative renal failure, which required dialysis.
Twenty-nine patients (4.7\%) required reoperation for bleeding. One patient $(0.2 \%)$ developed sternal dehiscence. Five patients $(0.8 \%)$ postoperatively developed stroke.

Twenty-five patients (4\%) died; the leading causes of death were low cardiac output and sepsis with eventual multi-organ failure.

\section{DISCUSSION}

The mean age of the patients in our study was $36.9 \pm 12.9$ years, which was a little higher compared with the mean age of $33.8 \pm 10.5$ years in a similar study but with a smaller number conducted by Wenbin et al [Wenbin 2002]. This could be because we included rheumatic, degenerative, and ischemic cases.

In our technique for mitral valve replacement, the posterior leaflet was preserved in all cases except 2 instances. In those 2 cases, both leaflets were kept to get the best outcome with postoperative EF. Geha et al mentioned that mitral valve replacement in patients with dilated left ventricle is associated with adverse consequences on left ventricular systolic function, resulting from interruption of the annulus-papillary muscle continuity [Geha 2004].

Gaiotto et al utilized different techniques to preserve the annulus-papillary muscle continuity and reported improvement in the left ventricular ejection fraction and decrease in the end-diastolic and end-systolic diameters up to the third month of follow-up [Gaiotto 2007].

Roman et al reported that aortic valve replacement resulted in normalized left ventricular chamber size and mass in twothirds of the patients recruited in their study and favorable geometric outcome is associated with persistence or recovery of normal left ventricular function [Roman 1989]. This is concordant with the excellent result in our study.

In our series, 25 patients (4\%) died, and the leading causes of death were low cardiac output and sepsis with eventual multi-organ failure. The relatively low mortality rate in this study could be explained by the low age group with fewer co-morbidities and secondary organ dysfunction. Jiang et al, in a study with a total of 126 patients with severely dilated left ventricle who underwent mitral valve and/or aortic valve operation, reported a similar mortality rate of $3.17 \%$; half of the cases died because of multiple organ failure secondary to severe low cardiac output syndrome. The other half died of sudden ventricular fibrillation [Jiang 2010].

Wenbin et al also reported a similar mortality rate of $4.4 \%$ in a study, which included a smaller number of 45 patients [Wenbin 2002]. However, Zhang et al reported a higher mortality rate of $8.8 \%$ in a study, which included 102 patients with rheumatic giant heart disease (cardiothoracic ratio $>$ or $=$ 0.8) who underwent valve replacement [Zhang 1994].

Recently, Jae et al, in a study to identify the predictors of left ventricular functional recovery and its impacts on clinical outcomes in acute heart failure patients with newly diagnosed dilated cardiomyopathy, found that larger initial left ventricular size was one of the independent predictors of poor left ventricular functional recovery [Jae 2018]. 
We think proper preoperative preparation is critical to getting the best outcome, especially with regard to measuring ESR, CRP and ASO for patients younger than 25 years. This also will help exclude ongoing rheumatic activity and performing Dobutamine stress echo for patients with EF less than $30 \%$ to confirm the presence for myocardial reserve for valvular cases and myocardial viability for ischemic cases.

Moreover, the special protocol, which was adopted for redo cases including blood ready in the OR before sternotomy, defibrillation pads on the chest and femoral artery and vein exposure before sternotomy, helped us minimize the risk of redo surgery for these patients.

Finally, the good number and multi-center characteristics of our study enabled us to describe clearly the behavior of cases with hugely dilated left ventricles in different surgical interventions.

\section{LIMITATIONS}

The major limitation of this study is that it reported shortterm outcome. In the future, we plan to report the mid-term and long-term outcomes for these patients.

\section{CONCLUSION}

Mitral and aortic valve replacement in patients with hugely dilated left ventricle are safe operations with satisfactory outcomes, even when combined with other procedures and with good preoperative preparations like dobutamine stress echo and inflammatory markers when indicated, intraoperative preservation of posterior mitral leaflet, and meticulous postoperative follow-up in the ICU.

\section{REFERENCES}

Cho JY, Kim KH, Song JE, Kim JE, Park H, Yoon HJ et al. 2018. Predictors of Left Ventricular Functional Recovery and Their Impact on Clinical Outcomes in Patients With Newly Diagnosed Dilated Cardiomyopathy and Heart Failure. Heart Lung Circ Jan;27(1):41-49.

Gaiotto FA, Puig LB, Mady C, Fernandes F, Tossuniam CE, Pardi MM et al. 2007. Mitral valve replacement with chordae tendineae preservation, traction and fixation in end-stage dilated cardiomyopathy. Rev Bras Cir Cardiovasc Jan-Mar; 22(1):68-74.

Geha AS, El-Zein C, Massad MG. 2004. Mitral valve surgery in patients with ischemic and nonischemic dilated cardiomyopathy. Cardiology 101(1-3):15-20.

Jiang SL, Li BJ, Gao CQ, Ren CL, Wang Y, Cheng TT et al. 2010. Clinical analyses of cardiovascular operations in patients with severe dilated left ventricle. Zhonghua Yi Xue Za Zhi. Nov 16; 90(42):2999-3002.

Roman MJ, Klein L, Devereux RB, Kligfield P, Niles NW, Hochreiter C et al. 1989. Reversal of left ventricular dilatation, hypertrophy, and dysfunction by valve replacement in aortic regurgitation. Am Heart J Sep; 118(3):553-63.

Wenbin L, Jianqun Z, Qiwen Z, Baotian C, Xiufang X, Zhaoguang Z et al. 2002. Surgical treatment of mitral regurgitation associated with enlarged left ventricle. J Heart Valve Dis Mar; 11(2):181-4.

Zhang BR, Xu ZY, Zhu JL. 1994. Pathological classification and clinical implications on rheumatic heart disease with giant heart. Zhonghua Wai Ke Za Zhi. Jun; 32(6):333. 